\title{
LIN OSA
}

\section{Bruno Messerli}

Linosa war das Ziel einer Exkursion Ende Februar 1958 unter der Leitung von Herrn Prof. Dr. F. Gygax, Direktor des Geographischen Institutes der Universität Bern. Die vorliegende Arbeit stützt sich vor allem auf unseren Exkursionsbericht, im besonderen auf die Beiträge von H. Schwarz und E. Gutzwiller.

\section{EINLEITUNG UND LAGE}

Linosa liegt auf dem 36 . Breitengrad, $150 \mathrm{~km}$ westlich von Malta oder $170 \mathrm{~km}$ südsüdwestlich Agrigento. Sie gehört geographisch zu den pelagischen Inseln, unterscheidet sich aber geologisch deutlich von ihnen.

Während Linosa rein vulkanischen Ursprungs ist, sind die andern Inseln der Gruppe, Lampedusa und Lampione, aus Kalken und Tuffen der tunesischen Kreide-Tertiärtafeln aufgebaut. Noch im Miozän standen die Südapenninenzüge westwärts in Verbindung mit den Telliden Tunesiens, als deren erythräisch abgeknickte östliche Enden sie anzusehen sind. Sizilien, Malta, Lampedusa und Lampione gelten heute als Reste dieser Landbrücke in der südlichen Tethys. Im Pliozän erfolgte, wie in andern Gebieten der Tethys, eine Meerestransgression, begleitet von starken Krustenbewegungen, die bis ins Quartär hineinreichten und sich heute noch in Erdbeben und im Vulkanismus äußern. Im Diluvium stürzte die Landbrücke ein, entsprechend ist heute das Relief des Meeresboden durch Brüche, seichte Schelfrücken und Schwellen gekennzeichnet. Gerade die Straße von Malta entspricht einer Schwellenzone mit einem 324 bis $1230 \mathrm{~m}$ tiefen Graben zwischen Malta und Lampedusa. Aus diesem Graben erheben sich die Vulkaninseln Pantelleria und Linosa. Ihre Lage in verkchrsgeographischer Hinsicht wurde uns eigentlich erst richtig bewußt, als das Kursschiff von Sizilien, das in Porto Empedocle bei leichtem Abendwind ausgelaufen war, nach einer schweren Sturmnacht im Morgengrauen vor der Küste zu ankern versuchte. Vergeblich rangen einige Männer im Ruderboot gegen die hochgehende See, sinnlos waren die Bemühungen, an die verbindende Falltreppe des Schiffes heranzukommen. Regen und Wind peitschten drei lange Hornstöße ans Ufer, enttäuscht zogen die Einwohner ihr Boot vor der Brandung in Sicherheit, schwankend entschwand der kleine Dampfer Richtung LampedusaPantelleria-Trapani. Zweimal wöchentlich bedeutet dieses Kursschiff die Verbindung mit der Welt; wie schwach aber diese Verbindung ist, hatten wir in diesem Augenblick erlebt.

\section{ENTSTEHUNG UND AUFBAU}

Der Sockel der Insel baut sich aus einem mächtigen Schild von Basislaven auf, die fast überall an der Küste herausquellen und so die Insel vor allzu rascher Zerstörung durch Meeresbrandung schützen. Die ebenen Flächen landeinwärts sind größtenteils bedeckt mit Tuffen, Aschen und vulkanischen Verwitterungserden, Grundlage und Begrenzung für Gärten, Äcker und Wiesen. Über diesen bebauten Ebenen erheben sich neun Stratovulkane, teils in prachtvoller «klassischer» Ausbildungsart wie der Monte Vulcano, teils nur noch als stark zerklüftete Überreste eines bereits wieder zerstörten, aber ehemals mächtigen Kegels, wie die benachbarte Montagna di Levante.

Sedimente konnten nirgends entdeckt werden. Die vorhandenen Effusiva sind melanokrate Olivin- und Feldspatbasalte, Schlacken und gelbe bis graugrüne feinkörnige Tuffe. Baumnußgroße leukokrate mittelkörnige Plutonite wurden an der Montagna Rossa gefunden, eingebettet in dunkle Schlacken. Es lassen sich eindeutig zwei verschiedene Perioden vulkanischer Aktivität unterscheiden, welche durch auffallende makroskopische Verschiedenheit des gefördeten Materials gekennzeichnet sind. Die erste Periode ist durch Tuffe charakterisiert; bis zu 8o m mächtig liegen sie auf einem unsichtbaren, früheren Relief, so daß ein einheitliches System im Streichen und Fallen kaum festzustellen ist und die Ortsbestimmung eines «Urkraters» wohl immer hypothetisch sein wird. In einer zweiten Phase haben Basaltströme dieses weiche Gestein durchschlagen, der Tuffmantel wurde aufgwölbt und zerbrochen, die Lava ergoß sich ins Meer (Abb. 1). Auffallend sind die angebrannten Kontaktflächen der Tuffe überall da, wo sie von den jüngern Laven über- oder unterlagert worden sind. In der gleichen Phase hat man sich wohl auch den Auswurf der roten Schlacke zu denken, aus der sich die oberen Partien der eigentlichen Vulkanberge aufbauen (höchste Erhebung des Monte Vulcano mit $195 \mathrm{~m}$ ). Die Eruption dieser zweiten Periode waren mutmaßlich von großen tektonischen Bewegungen begleitet. Die Punta di Ponente ist ein. Beispiel dafür, wo wir heute tatsächlich den Querschnitt eines Vulkans zum Studium vor 


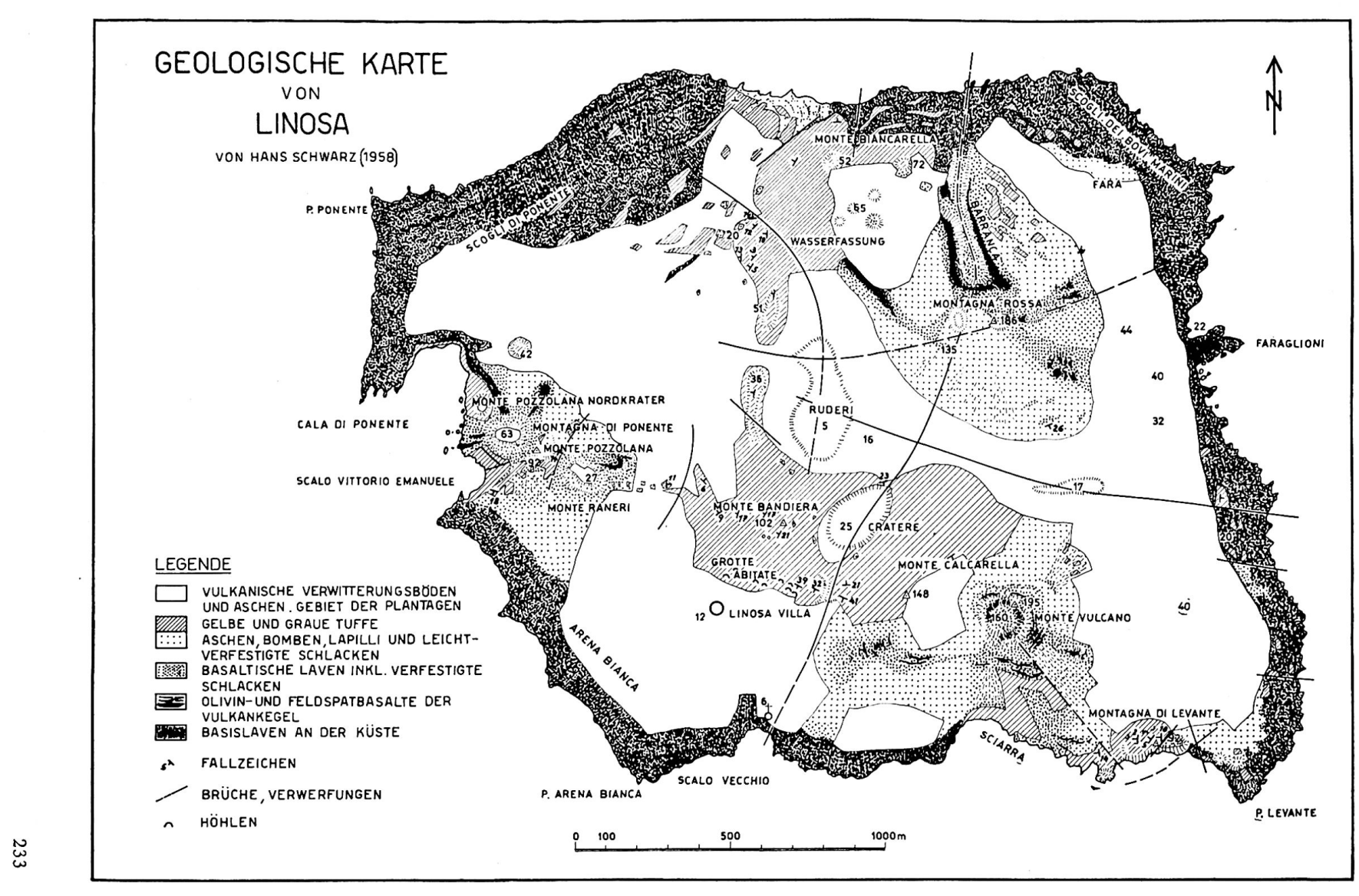




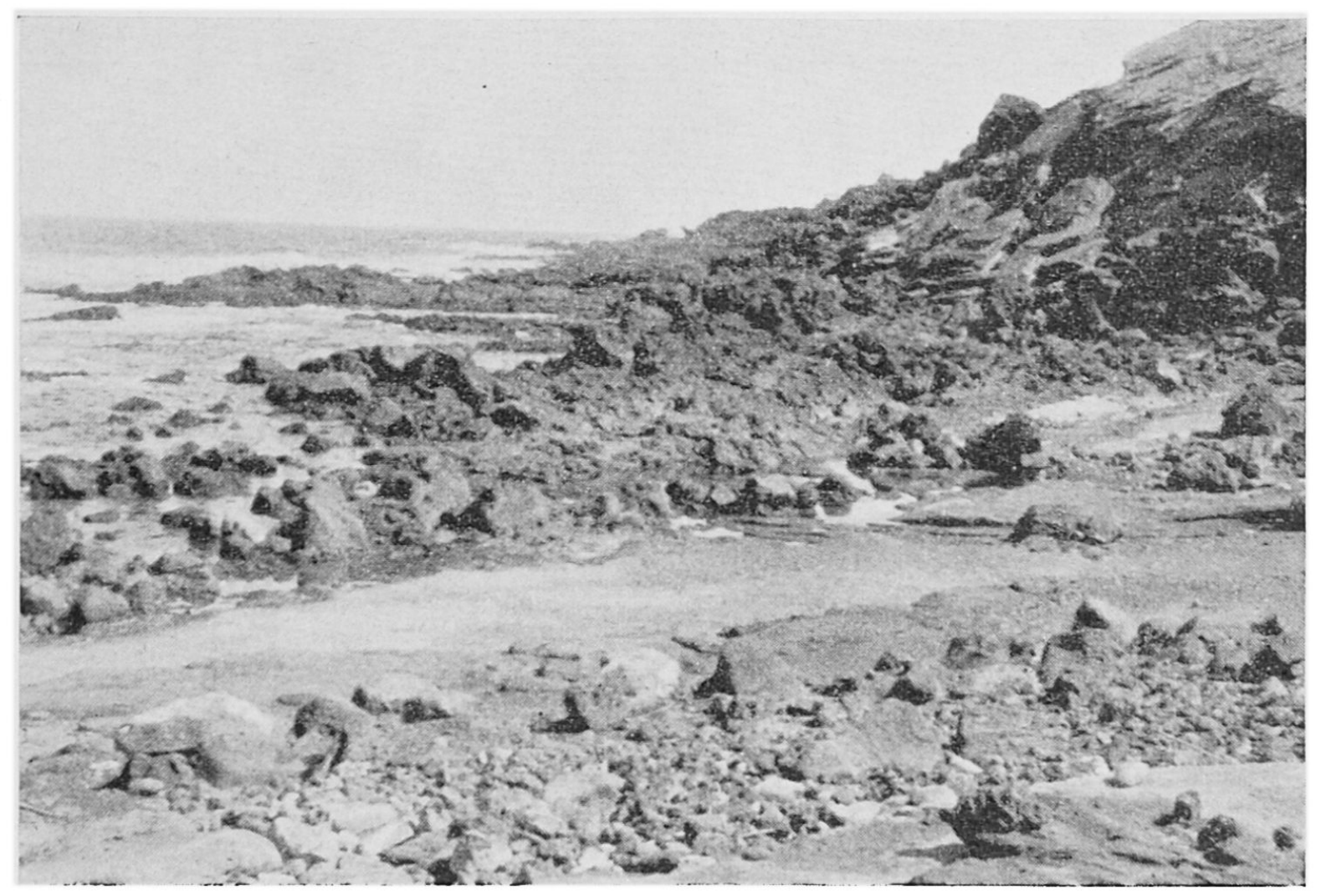

Abb. 1 Linosa, Küste am Mte. Biancarello: Lavaströme stoßen unter Tuff hervor.

uns haben, von dem ein Teil abgesprengt wurde oder entlang von Brüchen im Meer versank. Diese Bewegungen äußern sich aber auch als Klüfte und Verwerfungen in den Tuffschichten, sowie in einem größeren Bruchsystem, das die ganze Insel in verschiedenen Richtungen durchsetzt. (siehe geologische Karte). Diese geologischen Spezialuntersuchungen stehen nicht zusammenhanglos in unserer Gesamtarbeit. Sie verweisen auf die Ursachen der gegenwärtigen Bodenformen und auf die Art des Bodens, besonders weil die Humusschicht nur schwach ausgebildet ist. Die Morphologie begründet die natürliche Gliederung des Landes und bestimmt weitgehend die Siedlungsanlagen. Die Bodenarten scheiden deutlich das bewirtschaftete Land auf tuffig sandiger Grundlage vom mageren Allmendland auf den Schlacken der Steilhänge oder den Basalten der Küstennähe ( $\mathrm{Abb} .3$ und Karte 2).

\section{DAS KLIMA}

Vergeblich suchten wir nach genauen Temperatur- und Niederschlagsmessungen. Einst sollen genaue Untersuchungen gemacht worden sein, Ergebnisse davon waren aber nicht mehr aufzutreiben. Darum müssen wir uns mit einigen allgemeinen Feststellungen und Beobachtungen begnügen. Wir können kaum vom Mittelmeerklima im Schulbuchsinn sprechen. Die Insel liegt infolge ihrer Lage auch im Winter an der Grenze der Einflußsphäre des Subtropenhochs, was geringere Niederschläge als in Sizilien bedeutet. Auch die Temperaturen sind durchschnittlich höher als in Agrigent. Die Messungen von Malta entsprächen also den Verhältnissen in Linosa besser als etwa die Ergebnisse von Catania. Als eine Besonderheit muß gelten, daß die Vulkane zu wenig hoch sind, um niederschlagbringende Staulagen zu erzeugen (ganz anders Stromboli mit $926 \mathrm{~m}$ oder Salina mit $962 \mathrm{~m}$ ). Ferner ist die Humusschicht so dünn, daß die Hitzeausstrahlung der Schlacken und Basalte in den Sommermonaten wesentlich zum oftmaligen Absterben der Vegetation beiträgt. Der Wind ist ein ständiger und oft heftiger Gast auf der Insel; «Isola del vento» sollte sie eigentlich heißen, belehrte uns ein alter Bauer. Gerade in den Februarstürmen, durch die wir entgegen unserem wohlüberlegten Programm von der Umwelt abgeschnitten wurden, haben wir die Bedeutung des 
Windes erst richtig erkannt. Die hochaufspritzenden Wasserfontänen der Brandung reißt er mit sich fort, überspritzt die ungeschützten Felder, so daß die Vegetation oft durch die Versalzung zu Grunde geht; oder er scheuert wie ein Sandstrahlgebläse in den weichen Tuffen eine eigene Formenwelt heraus. Die Natur ist auf Linosa so dominant, daß die Kulturlandschaft ihr durchaus untergeordnet erscheint.

\section{GESCHICHTLICHES}

Was wir an Überlieferungen und Dokumenten zusammentragen konnten, ergibt in chronolologischer Reihenfolge ein eigenartig zerhacktes Bild. Auf der Suche nach der frühesten Besiedlung stoßen wir auf eine seltsame Nachricht, die im Dorf von Mund zu Mund geht. Man raunt ehrfurchtsvoll von einer versunkenen Stadt, $200 \mathrm{~m}$ von der Küste entfernt. Die Frage, ob es sich dabei tatsächlich um eine versunkene griechische oder römische Siedlung handelt oder lediglich um ein Schiffswrack, soll in der nahen Zukunft durch Taucher und Archäologen geklärt werden; im Augenblick wirft eine gehobene Amphore und das widerspruchsvolle Rätselraten der Bewohner einen mythischen Glanz auf die Geschichte der Insel. Skelettfunde werden von den einen als Sarazenen, von andern blo $B$ als Piraten der letzten Jahrhunderte gedeutet. Mit den Jahren 183o-1840 beginnen endlich eindeutige Tatsachen zu sprechen: Die staatlich organisierte Besiedlung des verwaisten Eilandes setzt ein. Dieses zusammenhanglose, phantasiegeschmückte Geschichtsfragment zeigt erneut die Isoliertheit der Insel und ihrer jeweiligen Bewohner. Nie konnte dieses Land in kulturgeschichtlicher Beziehung eine Rolle spielen, seine Lage war zu isoliert, sein Aufnahmevermögen zu klein, sein Boden zu arm.

\section{HAUS UND SIEDLUNG}

Vor 5 Generationen hatten sich 16 Siedler mit ihren Familien hierher gewagt, nachdem ihnen die Regierung eine Unterstützung zugesagt hatte. Was die Großväter damals alles erlebten, wissen uns die Ältesten im Dorfe noch genau zu erzählen. In den Höhlen der nahen Tuffelsen vegetierten sie am Anfang, zusammen mit ihrem Vieh, bis der Staat im Laufe der zweiten Generation die Häuser bereitgestellt hatte. Die Anlage der Siedlung erklärt sich allein aus der staatlichen Planung und Ausführung: Breite und gerade Hauptgasse, Konzentrierung von Kirche, Schule, Gemeindehaus,

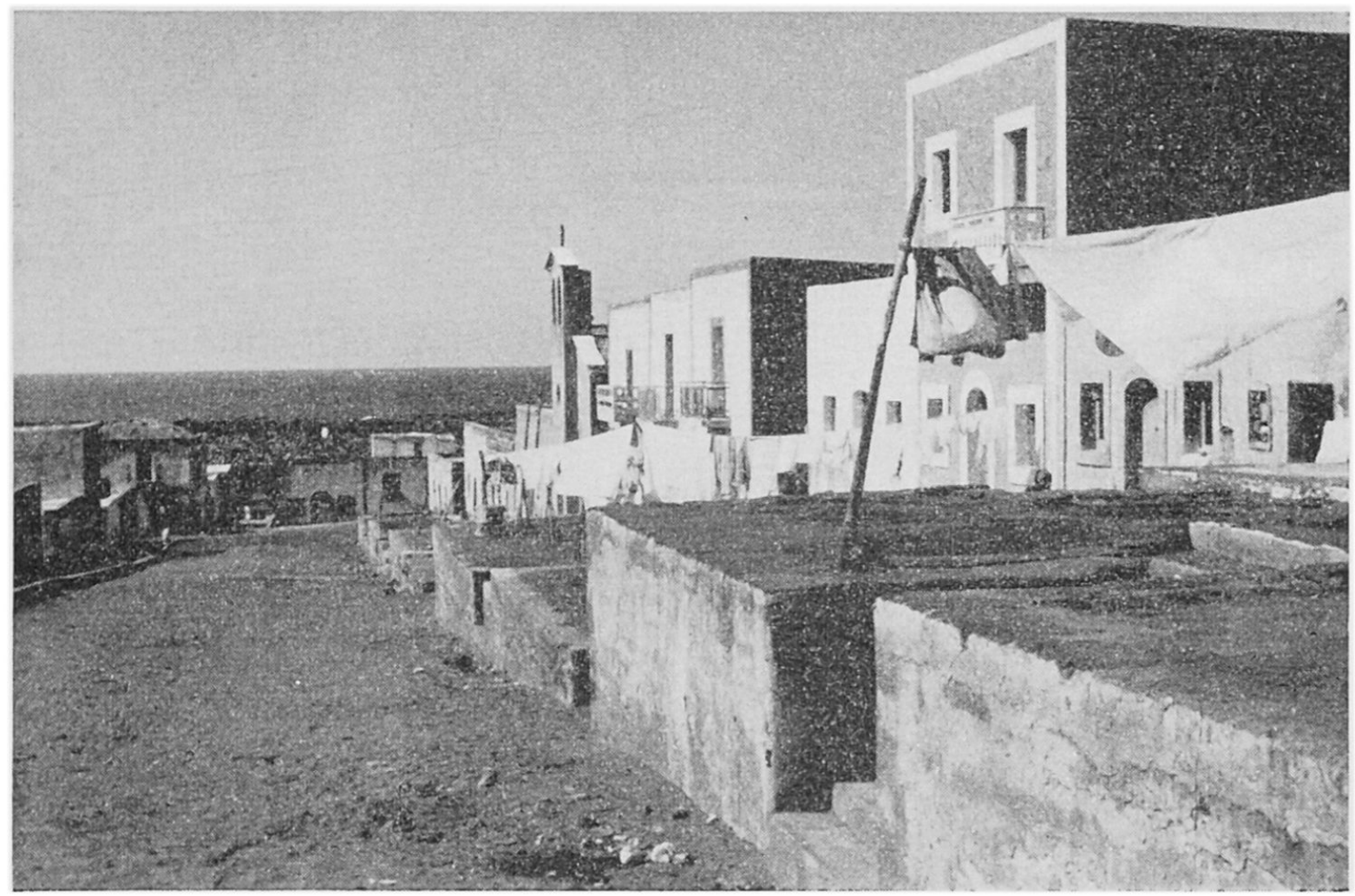

Abb. 2 Linosa, Via Vittorio Emanuele 


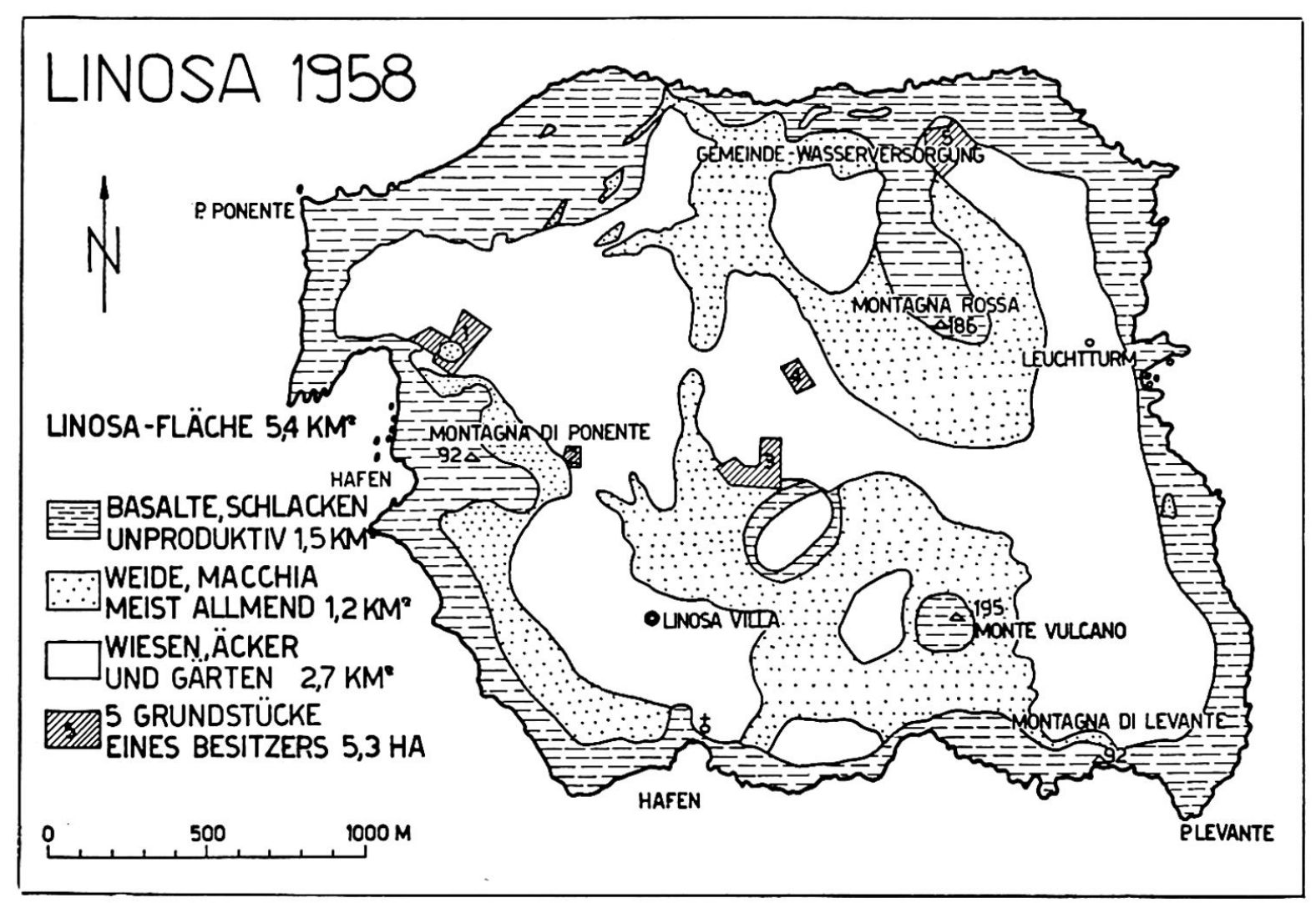

Karte 2

Post, Arzt und Händler, regelmäßige und gerade Seitengassen. Strenge Bestimmungen werden dieses einheitliche Bild der Siedlung auch in Zukunft wahren, zum Beispiel die Anlage der Straßen, vor allem die breite Via Vittorio Emanuele (Abb. 2). Erst in der neuesten Zeit hat sich eine Auflockerung ergeben durch privaten Häuserbau an der Peripherie des Dorfes und den Bau von Landhäusern auf den entfernteren Feldern. Aber auch diese zeigen nicht den erwarteten individuelleren Ausbau. Einmal ist das Flachdach eine Grundvoraussetzung im Bauplan des Hauses. Ergänzt durch ausgemauerte Flächen neben dem Hause, bilden sie mit 9-16 $\mathrm{m}^{2}$ das «Einzugsgebiet» des begehrten Regenwassers. Von da läuft es in die Zisternen; die größten der Insel haben wir mit einem Inhalt von $64 \mathrm{~m}^{3}$ berechnet. Ein weiterer Faktor der im Häuserbau mitspielt, ist die Armut der Bewohner. Diese besitzen höchstens 2-3 Räume, wobei die Innenausstattung zum Teil erst noch fehlt. Diese Bedingungen bestimmen jeden Bauplan mehr oder weniger zum voraus, und überdies würde das ungeschriebene Gesetz der Tradition jeden feudaleren Ausbau strengstens verbieten. Mit diesem gegenseitigen Angleichen, mit dem Ausschalten des Individuellen verfolgen wir bereits einen Wesenszug, der uns bei der Beschreibung des Menschen noch besonders auffallen wird.

\begin{tabular}{lrrrr} 
BEVÖLKERUNGSBEWEGUNG & \multicolumn{3}{c}{ KULTTURFL̈̈CH } \\
& 1842 & 1880 & 1922 & 1958 \\
\cline { 2 - 5 } Familien & 16 & 38 & 70 & 133 \\
Einwohner & 70 & 170 & 250 & 428
\end{tabular}

Der Bevölkerungszunahme, die sich durch diese wenigen Zahlen dokumentiert, ist eine unausweichliche Grenze gesetzt: $5,4 \mathrm{~km}^{2}$ Land stehen den Bewohnern zur Verfügung. Davon entfallen auf:
Unproduktives Land
$1,5 \mathrm{~km}^{2}$ oder $28 \%$
Weide, Macchia, meist Allmendland
$1,2 \mathrm{~km}^{2}$ oder $22 \%$
Kulturland
$2,7 \mathrm{~km}^{2}$ oder $50 \%$ 
Während die ersten Ansiedler mehr als genug Land zur Kultivierung vor sich sahen, erhält heute eine Familie im Durchschnitt bloß noch 3 ha, wovon ein Drittel mageres Weidland ist. Der ertragsarme Boden und die ungünstigen klimatischen Bedingungen schließen jedoch jeden Vergleich mit einem schweizerischen Kleinbetrieb aus. Dazu tritt eine zunehmende Auflösung der Besitzungen durch die Erbteilung. Auf dem Felde arbeiten heißt hier ständig herumwandern. Und diese Realteilung geht noch weiter; jedes Stück Land wird im Erbgang durch Anzahl der Söhne und Töchter geteilt, gleichgültig ob der Sohn irgendwo in Italien in einer Fabrik arbeitet, oder ob er es selber bewirtschaftet. Immer kleiner werden die Felder, immer enger rücken die Zäune aus Feigenkakteen aneinander. Die Bevölkerung wächst ständig, die Bodenfläche bleibt unabänderlich gleich. Vermehrte Auswanderung wird wohl in Zukunft nicht zu umgehen sein; nur zu gut haben wir den Wunsch nach Arbeit in der Schweiz verstanden. Vorläufig aber ist die Tradition und die Kraft der Lebensgemeinschaft aller Bewohner noch zu stark, sie werden hier geboren, sie teilen miteinander, was sie haben und sterben hier. Wie wird es aber in wenigen Jahren aussehen, wenn unvernünftige Touristen ihren Reichtum demonstriert und heimkehrende Auswanderer den Daheimgebliebenen unsere Lebensauffassung beigebracht haben? Werden wir dann die jetzige Atmospäre dieser Insel wiederfinden? Vorläufig geht das einzige Bestreben der Bewohner darauf aus, den Ertrag zu vergrößern, alles Wachstum sich zu nutze zu machen. Das großartigste Beispiel sind wohl die Fichi d'India, diese mancherorts wuchernden Feigenkakteen, vielfach als Unkraut verdammt, auf Linosa eine nicht wegzudenkende Nutzpflanze. In Hecken geordnet grenzen sie die Felder ab, schützen die Pflanzungen gegen den ständigen Wind und fangen die verderblichen Salzwasserverwehungen auf. Die alten Stämme ersetzen das fehlende Holz zum Feuern, aus den Früchten gewinnen die Frauen einen süßen Honig zum Brotaufstrich und das Wichtigste: Die gehackten Blätter bilden das einzige Futter für Kühe und Esel, wenn die Grasnarbe in der Sommerhitze versengt ist. Diese Kakteenreihen geben auch dem Flur-

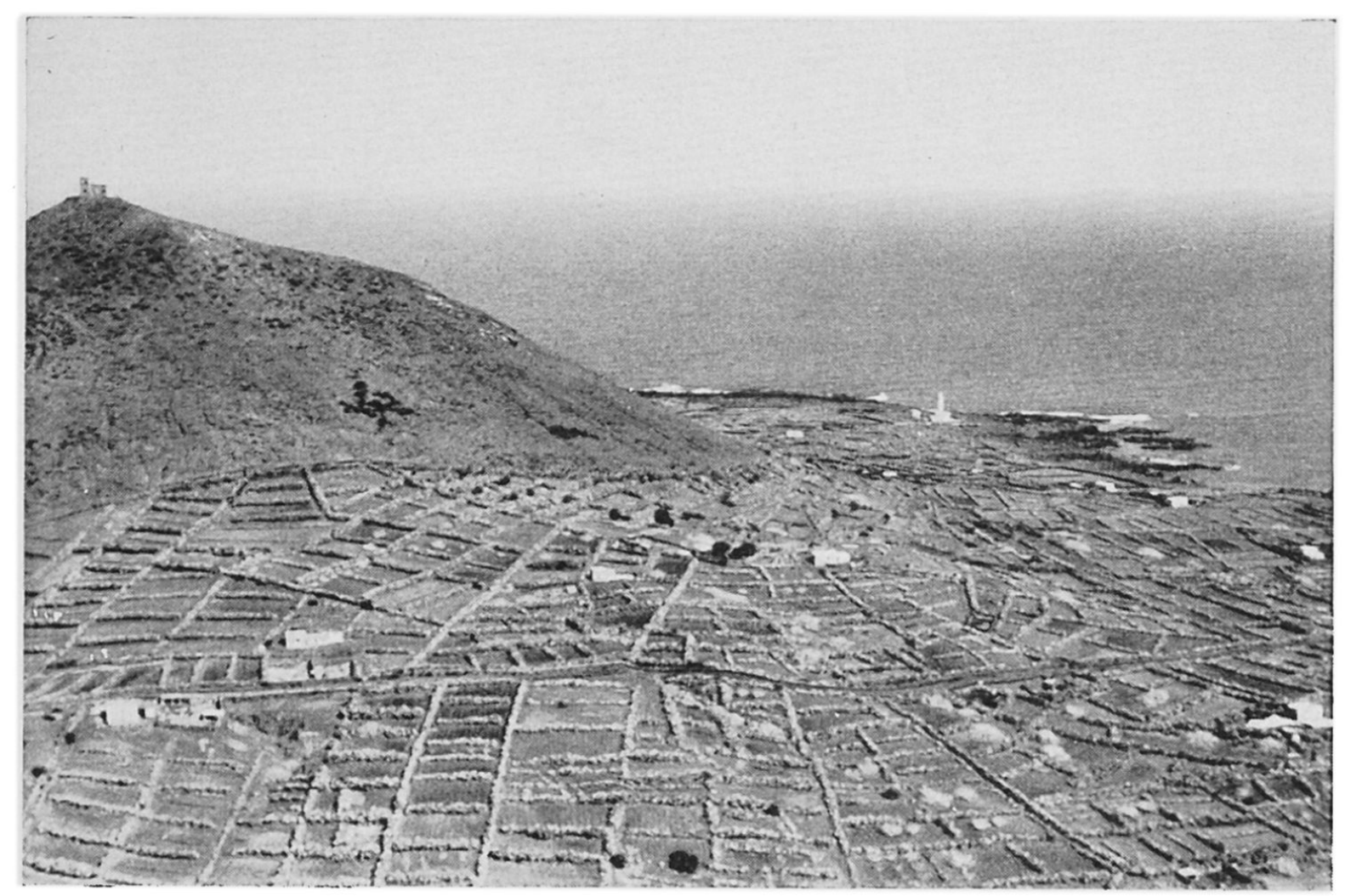

Abb. 3 Linosa: Küstenebene und Montagna Rossa von Mte. Vulcano aus. 
bild ein ganz eigenes Gepräge (Abb. 3). Dazwischen aber liegen infolge Erbteilung durcheinandergewürfelt, die Grundstücke der verschiedenen Besitzer. So hat zum Beispiel der Bauer Benedetto Ziardino fünf Parzellen auf der Insel verteilt (Karte 2).

Die folgende Tabelle gibt Aufschluß über seine Anpflanzungen:

\begin{tabular}{|c|c|c|c|c|c|c|c|}
\hline Bepflanzung & 1 & 2 & $\begin{array}{c}\text { Grundst } \\
3\end{array}$ & 4 & 5 & $\begin{array}{c}\text { Total } \\
\mathrm{m}^{2}\end{array}$ & $\begin{array}{l}\text { Antei } \\
\text { in } \% \text { des } \\
\text { Ganzen }\end{array}$ \\
\hline Reben & 1048 & - & - & - & - & 1048 & 1,975 \\
\hline Feigen/Reben & 455 & - & 2662 & - & 556 & 3673 & 6,930 \\
\hline Oliven'Reben & 827 & - & 2450 & - & - & 3277 & 6,177 \\
\hline Orzo/Gerste & 2985 & - & 1848 & 1790 & 4053 & 10676 & 20,124 \\
\hline Zuckererbsen & 1489 & - & 320 & - & 361 & $21 ; 0$ & 4,090 \\
\hline Tomaten & 1881 & - & - & - & 1315 & 3196 & 6,025 \\
\hline Gartenpflanzung & 493 & 500 & 2342 & - & 407 & $37+2$ & 7,053 \\
\hline Fichi d'IndialGras & 2958 & - & 9755 & 512 & 4142 & 17367 & 32,736 \\
\hline Gras & 2319 & - & - & - & 2754 & 5073 & 9563 \\
\hline Stallgehege & 337 & - & 150 & 188 & 569 & 1244 & 2,345 \\
\hline Unbepflanzt & 1312 & - & 270 & - & - & 1582 & 2,982 \\
\hline Total & 16104 & 500 & 19797 & 2490 & $1+157$ & 53048 & 100,000 \\
\hline Dazu gehören: & 1 & 2 & 3 & 4 & 5 & Im Dorf & Tota \\
\hline Häuser & - & 0,25 & - & - & - & 1 & 1,25 \\
\hline Stallgehege & 2 & - & 2 & 1 & 2 & - & 7 \\
\hline Zisternen & 1 & 0,25 & 1 & 1 & 1 & 1 & 5,2 \\
\hline Rebstöcke & 367 & - & 488 & - & 63 & - & 918 \\
\hline Feigenbäume & 23 & - & 33 & - & 6 & - & 62 \\
\hline Olivenbäume & 22 & - & 27 & - & - & - & 49 \\
\hline
\end{tabular}

Der Linosabauer richtet seinen Anbauplan nach zwei Gesichtspunkten aus, einerseits nach den Bedürfnissen der Familie, anderseits aber nach seinem Haupterwerbszweig, der Viehzucht. Diese Ausrichtung führt notwendigerweise zu einer gewissen Fluraufteilung, die einzelnen Anpflanzungen stehen zueinander und zum gesamten Besitz in einem bestimmten Verhältnis, was die folgende graphische Darstellung deutlich zeigt.

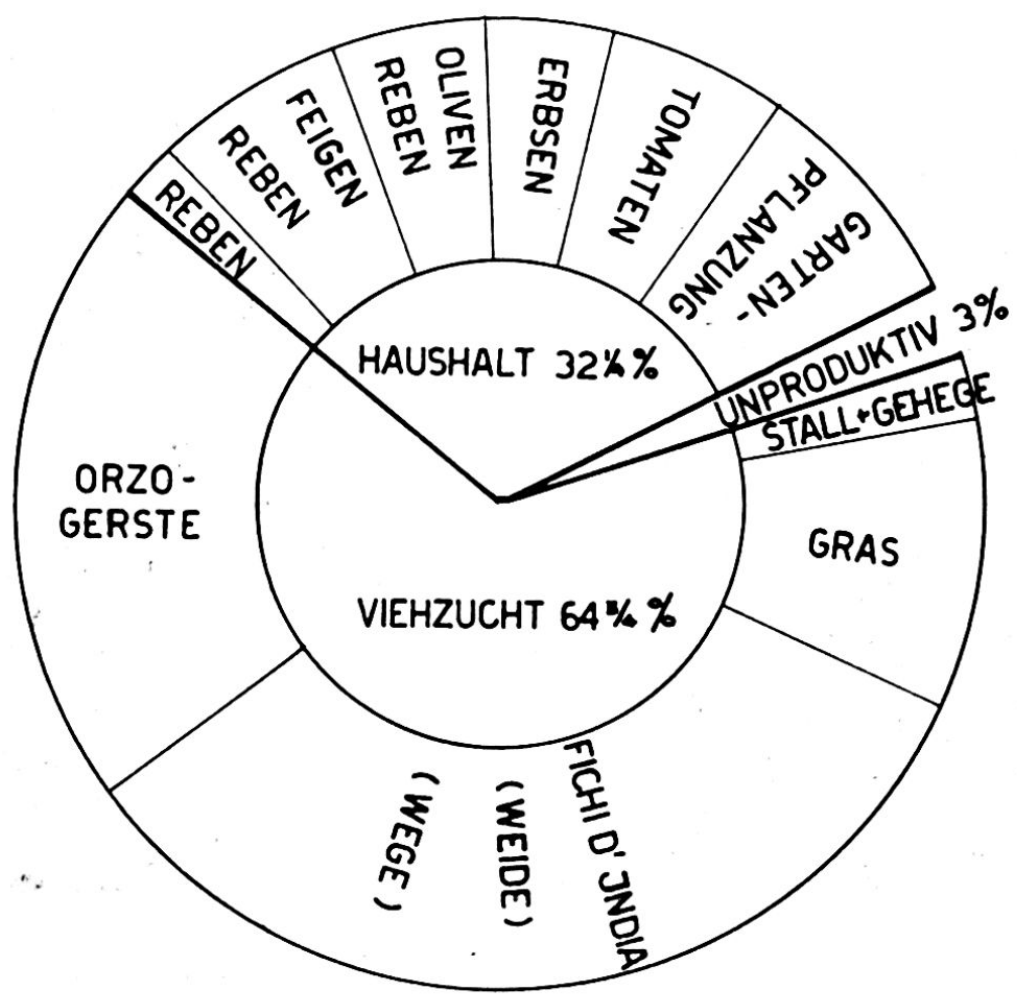

Einige initiative Bauern versuchen immer wieder Neuanpflanzungen. Stolz erhebt sich eine Dattelpalme am geschütztesten Ort der Insel, ihre Früchre gelangen aber nie zur Reife. Ein einziges Orangenbäumchen mit herrlichen Früchten ist eine kleine Sensation. Wenn wir aber bedenken, daß drei $\mathrm{Zi}$ sternen sein Wachstum gewährleisten müssen, dann sehen wir auch hier wieder die Grenzen menschlicher Initiative.Überhaupt zeigt das ganze Vegetationsbild, wie die Natur Grenzen setzt, die der Mensch nicht überschreiten kann. Einerseits ist es das fehlende Wasser, das alles 
Leben aufhält. Allerdings läßt man in echt südlicher Mentalität eine vom Militär gebaute Gemeindeversorgung zu Grunde gehen, weil man sich eben auf das Wasserschiff vertröstet, das im Notfalle von Messina kommend die leeren Zisternen im Dorfe auffüllt. Anderseits zwingt das Klima zu schnellem Wachstum. In zwei Wochen werden die kahlen weißen Äste der Feigenbäume hinter üppigem Grün versteckt sein und im Juli liest man schon die ersten Trauben ab. Dann aber folgt die Zeit des Stillstands, Trokkenheit, Dürre, "Isola nera".

\section{DIE MENSCHLICHE LEBENSGEMEINSCHAFT}

Armut kennzeichnet die soziale Lage der Bevölkerung und spiegelt sich in der eigenartigen Einförmigkeit der beruflichen Struktur. Jeder strebt nach totaler Selbstversorgung, immerhin muß er sich Mehl, Öl, Salz, Seife, Zucker und Petrol beim Händler erstehen. So kommt es vor, daß ein Bauer bis zu 1000 Schweizerfranken verschuldet ist. Die Abzahlung wird nur durch die Aufzucht und den Verkauf von Jungvieh möglich. Für 7-8 Monate alte Tiere können ungefähr 150000 Lire gelöst werden, ein Esel dagegen gilt nur 30000 Lire. Dazu kommt für die meisten Männer ein kleiner Nebenverdienst, sei es als Fischer, als Säumer für den Warentransport HafenDorf oder als Ruderknecht bei Ankunft und Abfahrt des Kursschiffes. Die beiden letzteren sind von der Schiffahrtsgesellschaft bezahlt. Von eigentlichen Gewerben können wir kaum sprechen, abgesehen von 2 Schneiderinnen, 2 Schreinern und einem Korber. Aus dieser Eintönigkeit der Berufsstruktur fallen der Arzt, der Lehrer und der Polizist eher als fremde Bestandteile heraus. Ist es da verwunderlich, daß ein Kommilitone von uns mitten in der Nacht von seinem Gastgeber zur Geburtshilfe herbeigerufen wurde, währenddem man den staatlich angestellten Doktor ruhig zu Hause weiterschlafen ließ. Der Arzt ist eben etwas fremdes, er kommt aus einer andern Welt, auf die man auch sonst nicht zählen kann; der Gast dagegen wird lieber ins Vertrauen gezogen, wenn er in die Familiengemeinschaft aufgenommen worden ist.

Diese wenigen Bemerkungen geben im Grunde genommen nur ein verschwommenes Bild von der Stellung der Bewohner. Wir können aber in Linosa auch nicht ein Dorf als Lebenszentrum von einer Agrarlandschaft unterscheiden, die beide zueinander in einer bestimmten Funktion stehen. Der Bauser wohnt selber im Dorf, er ist auf keinen marktwirtschaftlichen Austausch angewiesen, umso mehr als kein Gewerbe existiert und er nach möglichst großer Selbstversorgung und Selbständigkeit trachtet. Dorf und Land werden dadurch identisch. Die Organisation der Bevölkerung ist weder politisch noch wirtschaftlich, sie beruht viel mehr auf gefühlsmäßig verwandtschaftlichen Bindungen. Auf sich allein angewiesen, zusammengeschweißt durch die Naturgewalten, bilden die Linosaner eine Lebensgemeinschaft. In diesem Zusammenschluß findet der Einzelne seine einzige Hilfe. Äußerlich dokumentiert es sich dadurch, daß alle einander verwandt sind, innerlich aber zeigt sich ein auffallendes Angleichen und Anpassen aller an alle in Wesen und Gestalt. Das Individuelle, das Ausgefallene hat in dieser Gemeinschaft keinen Platz. Jeder kennt jeden, niemand würde es z.B. wagen, etwas zu stehlen. Der Schlechte hat keinen Platz in der Gemeinschaft, er kann sich nicht verstecken, jeder steht unter der absoluten Kontrolle des Ganzen. Darum sind Gefängnisse oder Verhaftungen hier etwas Nie-Gesehenes. Aber auch eine Zeitung wäre sinnlos; folgende Begebenheit mag es illustrieren: Am Strande begegnen wir einem Bauern, barfuß geht er ruhigen Ganges über die glasharten Basalte, denn Schuhe sind für die tägliche Arbeit unerschwinglich. Er sucht nach $\mathrm{Holz}$, das vom gestrigen Sturme angeschwemmt wurde. Wir bleiben vor einem Stück Palmenstamm stehen, aus Afrika werden es die Fluten herangetragen haben. Warum greift er nicht nach diesem Reichtum? «Weil es schon jemandem gehört.» Warum gehört es jemandem? Der Bauer lächelt verlegen, er weiß es einfach. Lange geht es, bis wir endlich begreifen, was er meint. Ein anderer hat diesen Palmstrunk gefunden, am Abend weiß jedermann im Dorf, daß dieses Stück 
Holz bereits einen Besitzer hat. So funktioniert die mündliche Dorfpost bis zu Kleinigkeiten. Könnte man den Begriff Lebensgemeinschaft besser definieren als mit diesem scheinbar nichtssagenden Erlebnis. Letztlich beruht auch die unvorstellbare Gastfreundschaft auf dieser Einstellung. Daß unsere Zelte in einer Sturmnacht weggefegt wurden, alarmierte das ganze Dorf mit einem Schlage. Gastlich wurden wir in ihren Häusern aufgenommen, mehr als ihre Armut zuließ, haben wir empfangen. Indem wir ein wenig in ihr Dasein hineinwuchsen, wurde uns alles gegeben, was ihnen gehört; wir wurden für einen kurzen, aber unvergeßlichen Augenblick ein Glied ihrer Lebensgemeinschaft.

Dieser Abriß aus einer größeren Arbeit mag zeigen, wie vielfältig Ursachen und Wirkungen im Erscheinungsbild dieser Insel ineinanderspielen, obschon wir doch von einer Landschaft einfachster Ordnung sprechen könnten. Ein Unterschied zur unsrigen hebt sich deutlich heraus. Es ist die unmittelbare Dominanz der Natur, nach deren sich alles Leben ausrichtet. Durch unsere Detailarbeit, die feinern Zusammenhänge zu sehen und hinter den Erscheinungen der Ursachen zu erkennen, das war und wird ein Ziel unserer Arbeit und unserer Exkursionen sein. In diesem Sinne bleibt uns Linosa eine nachhaltige, persönliche und belehrende Erinnerung und dafür gilt unser Dank besonders Herrn Prof. Dr. F. Gygax

\section{LINOSA}

Nel febbraio di questo 1958, sotto l'esperta guida del Prof. Dott. GyGax, studenti di geografia dell'Università di Berna soggiornarono sull'isola di Linosa, per studiarne la costituzione fisica e le conseguenti particolarità. In un fasciolo di 150 pagine, riccamente illustrato da fotografie, schizzi e tabelle riguardanti la geologia e la vegetazione, essi delineano un quadro completo di quest'isola vulcanica del Mediterraneo, che pur distando ben $170 \mathrm{~km}$, fa parte della Provincia di Agrigento. Linosa: $5 \mathrm{~km}^{2}$ di tufi, lava, lapilli e basalti. Battuta dai venti, inondata di sole, corrosa del mare. Dal Mte. Vulcano $(195 \mathrm{~m})$ o dalla Montagna Rossa $(186 \mathrm{~m})$, la si domina tutta: uno scacchiere di campicelli circondati e suddivisi da siepi di fichi d'India, che limitano la proprietà e proteggono le diverse culture dal vento che soffia da mare a mare. Il frazionamento è dovutu alla ripartizione derivante della credità. Verdeggiante in inverno e primavera, arsa in estate ed autunno, non ha una sorgente, non un rigagnolo. Ogni goccia d'acqua, che non sia racolta di terrazzi di cemento dei tetti delle abitazioni o costrutti appositamente. Riuniti in una sola località : Linosa Villa, 428 abitati in 133 famiglie. Un'unica grande famiglia di gente onesta, buona e generosa, associata nella dura lotta per l'esistenza, al di sopra di ogni individualismo ed egoismo.

\section{CHATILLON-SUR-SEINE}

\section{WILly MEYER}

Das Châtillonnais ist die nördlichste der geographisch so verschiedenen Landschaften Burgunds - dessen, was wir heute unter Burgund verstehen, im wesentlichen des Kernlandes der «Grands Ducs d'Occident», der vier Valois, Philipps des Kühnen, Johanns Ohnefurcht, Philipps des Guten und Karls des Kühnen. Das Châtillonnais gehört zum Departement der Côte-d'Or, als dessen Nordzipfel. Es ist ein wenig fruchtbares Gebiet. R. H. Francé schrieb einmal: «Daß Sandstein wasserdurchlässig sein kann, wird man noch leicht glauben; daß aber auch der Kalk Wasser durchsickern läßt, davon muß man sich schon überzeugen.» Im Châtillonnais kann man sich davon überzeugen. In dieser Zone von Felsplateaus, die von dürren Tälern durchschnitten wird, ist der Boden trocken; lediglich dank dem vielen Regen ist dennoch Waldbestand möglich. Wie in der Gegend des provençalischen Vaucluse gibt es auch hier unterirdisch reichlich Wasser. Die Douix, die in Châtillon der Seine zufließt, bildet eine «source vauclusienne». Das Châtillonnais war früher durch seine zahlreichen Schmieden bekannt, wie sie zum Beispiel die Cisterziensermönche betrieben. Sie sind größtenteils verschwunden, von anderen, moderneren Fabrikationsmethoden verdrängt. Ebenfalls der Vergangenheit gehört die Merinozucht an, die in früheren Jahrhunderten Châtillon- 